\title{
Development of a Compact and Low-Cost Weather Station for Renewable Energy Applications
}

\author{
Jose A. Salgado ${ }^{1}$, Miguel C. Feio ${ }^{2}$, Luis M. Silva ${ }^{2}$ \\ Vitor Monteiro ${ }^{2}$, Joao L. Afonso ${ }^{2}$ and Jose A. Afonso ${ }^{1, \#}$ \\ ${ }^{1}$ CMEMS-UMinho Center, University of Minho, Guimarães, Portugal \\ ${ }^{2}$ ALGORITMI Research Centre, University of Minho, Guimarães, Portugal \\ \#jose.afonso@dei.uminho.pt
}

\begin{abstract}
This paper describes the development of a weather station integrating several sensors which allows the measurement and data storage of the following environmental parameters: solar irradiance, temperature, humidity, wind speed, and wind direction. The collected data is later transferred to a mobile device, where it is stored in a database and processed in order to be visualized and analyzed by the user. For such purpose, a dedicated mobile app was developed and presented along the paper. The weather station also integrates small solar photovoltaic modules of three different technologies: polycrystalline, monocrystalline and amorphous silicon. Based on that, the weather station also collects information that may be employed to help the user in determining the most suitable solar photovoltaic technology for installation in a particular location. The developed system uses a Bluetooth Low Energy (BLE) wireless network to transfer the data to the mobile device when the user approaches the weather station. The system operation was validated through experimental tests that encompass all the main developed features, from the data acquisition in the weather station, to the visualization in the mobile device.
\end{abstract}

Keywords: Weather Station, Solar Photovoltaic Modules, Bluetooth Low Energy, Mobile App.

\section{Introduction}

As the world population increases, the demand for energy grows with it. Fossil fuels like coal, oil and natural gas still supply $80 \%$ of the energy demand. However, the environmental effects are a major problem associated with these sources of energy, due to the $\mathrm{CO} 2$ emissions [1]. Therefore, there is a need to focus on energy sources as optimal long-term solutions.

In this context, solar energy is one of main renewable energy sources that has been considered to tackle this problem. A total of $1.8 \times 10^{11} \mathrm{MW}$ of solar power is received by the Earth at instant [2]. Despite not covering all energy consumption needs, solar energy has the potential to cover a significant share of it. Photovoltaics (PV) is one of the methods to convert solar energy into electricity, and this technology has had a 
significantly improvement in the last years in terms of efficiency. It is based on semiconductors materials that exhibit the photovoltaic effect to convert the sunlight into electrical energy. These semiconductors are mainly made of silicon, which has a large availability in the Earth's crust. In terms of environmental effects, PV generates no pollution and its greenhouse gas emissions are non-existent. It also provides great scalability, meaning that its applications can range from few milliwatts to several hundreds of megawatts. However, the main disadvantage is that the production of energy is restricted to a range of hours during the day, which many times does not match the peak energy demand hours [3].

Concerning solar photovoltaic technologies, there is a variety of options. The most suitable choice will depend on the location and the scale of application. Crystalline silicon is the most prevalent material for solar photovoltaic modules. Its working principle is based on the concept of the p-n junction. Various sub-types of crystalline silicon materials can be used to develop solar photovoltaic modules. The most popular ones are monocrystalline silicon and polycrystalline silicon. Monocrystalline silicon is more efficient, but generally more expansive than other types. Polycrystalline are less efficient, but less expensive than the monocrystalline silicon ones, so they are the most common type used. Thin-film technologies are also commonly used to develop solar photovoltaic modules. Currently, the most used thin-film technology is the amorphous silicon. Despite producing less energy when comparing to crystalline technologies, it has the advantage of being flexible, which can be useful in some applications [4].

To determine the best solar photovoltaic technology to be used in a given location, the information about the weather conditions in that place is very important. The local temperature, solar irradiance, and even wind speed and direction, are some factors that have impact on the energy produced by solar photovoltaic modules, and that impact can be different for different technologies [5].

Concerning related work, in [6] a system to monitor the performance of solar panels using a low-cost Wireless Sensor Network (WSN) is proposed. Solar irradiance, and temperature, as well as the current and voltage from the solar panels were the measured variables. In this case, the collected data was continuously transmitted to a nearby personal computer using ZigBee. In [7] a remote data logger for weather forecasting based in a GPRS (General Packet Radio Service) connection was developed. The data gathered was stored locally in a SD (Secure Digital) card. The File Transfer Protocol (FTP) was used to transmit the collected data to a remote server. Every time the data logger receives a report with the measured parameters, it creates a new text file in the SD card and sends the file to the remote server. The GPRS connection requires a cellular device with a SIM (Subscriber Identification Module) card, meaning associated costs with the data transmission. In contrast, this paper proposes an innovative solution, where the data is stored locally and transferred to user device using a short-range wireless network when he approaches the weather station, which avoids the costs associated with data transfer.

As main contribution, this work presents the development of a compact and lowcost weather station to measure multiple environmental parameters. The collected data is later transferred to a smartphone, where it is stored and displayed to the user 


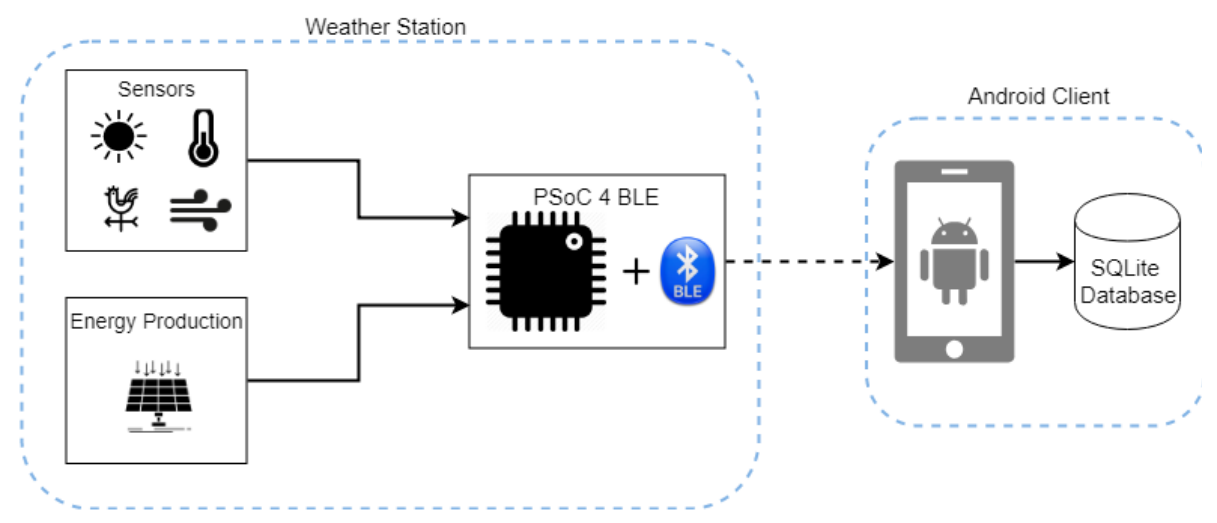

Fig. 1. Architecture of the developed system.

through a developed mobile app, specially dedicated for such purposes. The developed weather station prototype also integrates small solar photovoltaic modules of three different technologies: polycrystalline, monocrystalline and amorphous silicon. The aim is to allow correlating the collected environmental data to the power production of the three different solar photovoltaic technologies, in order to determine which technology is the most suitable for use in a particular location.

For convenience and easiness of access, the data is transferred to the user's mobile device using a wireless connection instead of cables, since the weather station may be installed in a hard-to-reach location. The developed system uses Bluetooth Low Energy (BLE) for the wireless data transfer due to native integration in mobile devices as well as its low energy consumption [8]. An alternative would be the use of an IEEE 802.11/Wi-Fi wireless network [9], which provides higher data rates, but with higher energy consumption. Other wireless network alternatives, like LoRa [10] and ZigBee [11], were excluded due to their lack of native support in smartphones and lower data rates.

\section{Developed System}

This section describes the development of the different components of the proposed weather station. Multiple sensors were used to allow the measurement of the following local weather parameters: solar irradiance, temperature, humidity, wind speed, and wind direction. The developed system is also able to measure the energy generated by three different solar photovoltaic technologies, in order to correlate their power production with the measurements of the weather sensors.

The architecture of the developed system, represented in Figure 1, is composed by two main parts: a weather station and an Android client. The data collected by the weather station is processed and stored locally in a SD memory card. When requested by the Android mobile app, the data is transferred to the mobile device using BLE, where it is stored in a local SQLite database and may be visualized by the user. 


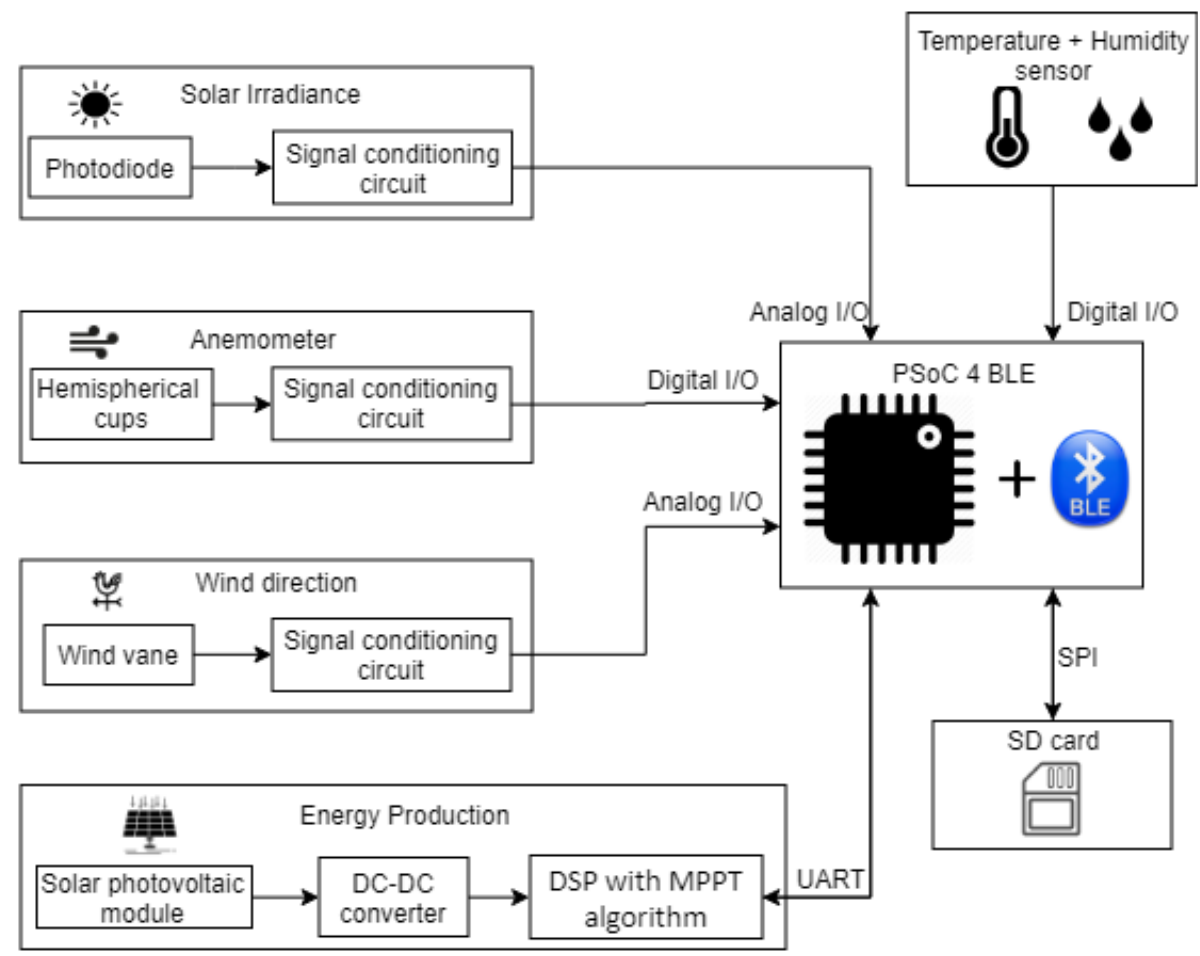

Fig. 2. Block diagram of the hardware used in the data acquisition process.

\subsection{Data Acquisition and Storage}

The block diagram of the data acquisition hardware implemented in the weather station is presented in Figure 2. The data acquisition and processing (as well as the wireless communication with the Android client) is performed by a PSoC 4 BLE module [12], from Cypress Semiconductor. This module, used in the developed system, includes an ARM Cortex-M0 32-bit microcontroller with integrated Bluetooth 4.2 protocol stack. It contains blocks for serial communication via UART (Universal asynchronous receiver/transmitter), SPI (Serial Peripheral Interface), or I2C (Inter Integrated Circuit). It also provides an ADC (Analog to Digital Converter) with a maximum resolution of 12-bits, four programmable operational amplifiers and four TCPWM (Timer Counter Pulse Width Modulator) blocks, which can be used for timers, counters or PWM generators.

The firmware of the PSoC 4 BLE microcontroller was programmed in $\mathrm{C}$ using the PSoC Creator IDE (Integrated Development Environment), which is also provided by Cypress. During the development phase, the PSoC 4 BLE module was attached to a development board for programming and debugging. Both of these boards are supplied in the CY8CKIT-042-BLE-A kit [12]. 


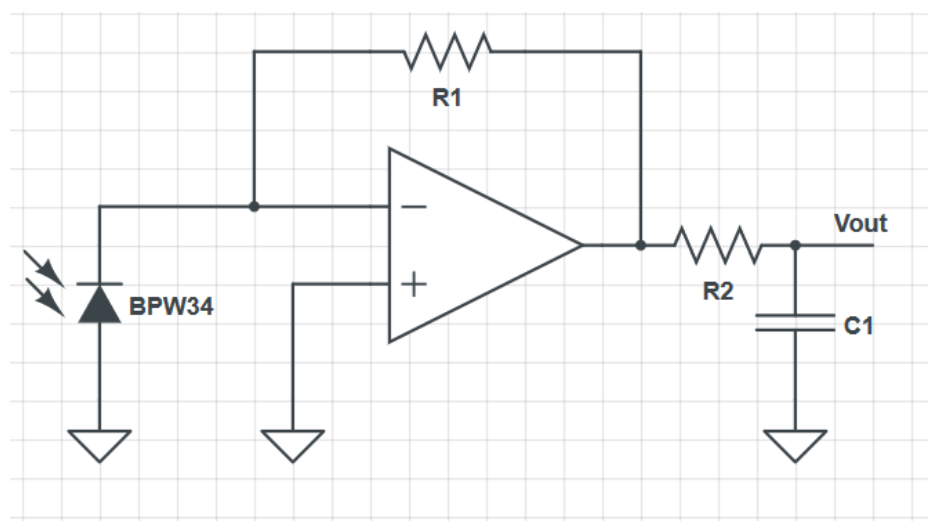

Fig. 3. Circuit used for measuring the solar irradiance.

The photodiode BPW34 [13] was used to measure the solar irradiance. This sensor was chosen taking into consideration the minimum and maximum values for the solar spectral radiation wavelengths, the cost and availability. The signal produced by the photodiode is fed into a signal conditioning circuit to convert the current produced into a voltage that can be read by the ADC of the microcontroller. This circuit was implemented using a transimpedance amplifier, as shown in Figure 3. For this purpose, one of the programmable operational amplifiers integrated inside the PSoC 4 BLE module was used in the circuit. For this purpose, the inputs and output of the operational amplifier were programmed to be connected to pins of the microcontroller using the PSoC Creator IDE. A low-pass filter was placed at the output of the operational amplifier to filter ambient noise. A precision pyranometer was used to correlate the output to provide a solar irradiation measurement in $\mathrm{W} / \mathrm{m}^{2}$.

The DHT22/AM2302 sensor was used to measure both the temperature and the humidity [14]. This sensor can measure temperatures from $-40^{\circ} \mathrm{C}$ to $80^{\circ} \mathrm{C}$ and relative humidity from 0 to $100 \%$. The output of this sensor is a 1-wire bus digital signal that can be read in a digital pin of the microcontroller. The data packet is composed of 40 bits, with 16 bits for the relative humidity data, 16 bits for the temperature data and 8 bits for checksum.

An anemometer is used to measure the wind speed. For this purpose, a magnet was attached to the rotational axis of the anemometer. The magnetic field created by this magnet is then detected by a Hall effect sensor [15], allowing to convert the mechanical movement into an electric signal. The output of this sensor is connected to a digital pin of the microcontroller, where it is processed in order to produce wind speed measurements in Rotations Per Minute (RPM).

An electronic wind vane was used to measure the wind direction. For this purpose, a magnet is attached to the rotational axis, and the magnetic field is then detected by a reed switch. A total of 8 reed switches are used for 8 different wind directions. Each reed switch is connected in series with a resistor of different value. Depending on the wind direction, a single reed switch will be closed. Another resistor at the end of the circuit forms a voltage divider with the resistor associated with the activated reed 


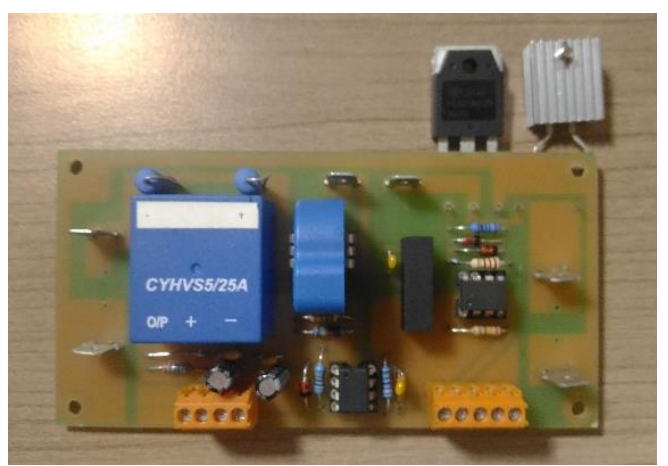

Fig. 4. Developed DC-DC converter for interfacing the solar photovoltaic modules.

switch, which means that for each wind direction, a different voltage value is obtained. The resulting voltage is then measured with the ADC of the microcontroller.

A DC-DC converter in a step-up topology was also developed, as shown in Figure 4 , to work in conjunction with the solar photovoltaic modules. Varying the value of the duty-cycle applied in the semiconductor $\mathrm{S}$, it is possible to adjust the operating power (sampling and switching frequency of $40 \mathrm{kHz}$ and $20 \mathrm{kHz}$ were considered, respectively). The converter is controlled by a MPPT (Maximum Power Point Tracking) algorithm implemented in a Digital Signal Processor (DSP) [16]. The objective is to measure the power produced by the three technologies of solar photovoltaic modules installed in the weather station, where the MPPT algorithm is responsible to guarantee that the solar photovoltaic modules are operating at the maximum power. A power resistor was used as load of the DC-DC converter. On the DC-DC converter input, a voltage and a current sensor were installed, and supported by a developed signal conditioning circuit, the current and voltage values are acquired by the ADC of the DSP (where is implemented the MPPT algorithm). The MPPT algorithm was digitally implemented in the development board LAUNCHXL-F28027F from Texas Instruments. It is important to note that in our approach, a single DC-DC converter was developed, but, based on an external circuit, it is connected sequentially to each solar photovoltaic module. The values obtained by this board are then saved and transferred to the PSoC board using the UART interface when required.

The Perturb and Observe (P\&O) MPPT algorithm was used, which is a trial and error algorithm, where the controller adjusts the duty-cycle while measuring the output power. This process is continuously performed until there is no more power increase. This method is the easiest to implement, but has the disadvantage to cause some oscillations in the power output; however, it is not critical for the purpose of the weather station presented in this paper.

To acquire the data from all the sensors, various peripherals were programmed in the PSoC module. A timer was programmed to establish the polling rate at which the data from the sensors are acquired. This value is set by the user in the Android mobile app. This timer functions in interrupt mode, activating a flag inside the ISR (Interrupt Service Routine). This flag is then verified in the main loop and, in case of being activated, the values are acquired from the sensors. To measure the solar irradiation 


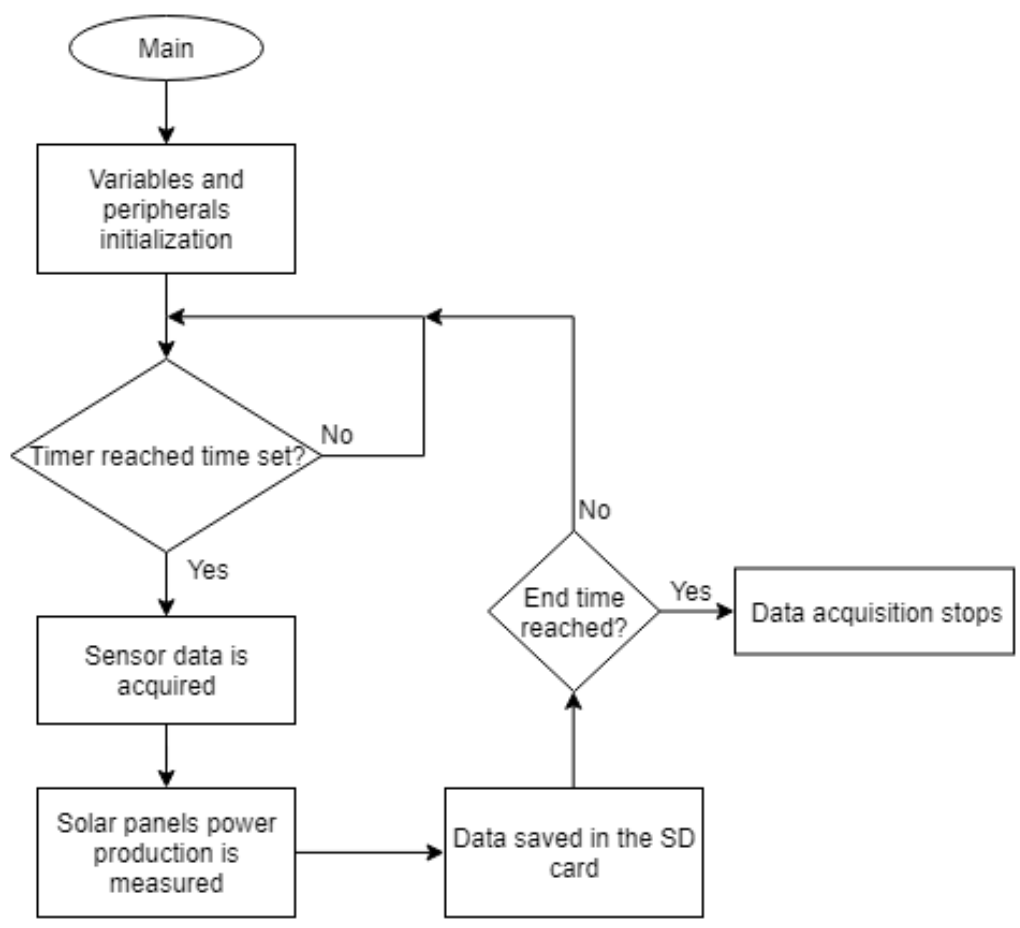

Fig. 5. Flowchart of the main function used for the data acquisition.

and wind direction, the ADC of the microcontroller was programmed with the maximum resolution of 12 bits, a sample frequency of $4 \mathrm{kHz}$ and a voltage reference of $5 \mathrm{~V}$. For measurement the wind speed, a counter was programmed in interrupt mode to count the amount of time for a rotation to be completed and then the value is converted to RPM.

The data from all the sensors is saved periodically in a text file corresponding to the day of acquisition. This process is done until it reaches the end time, defined by the user. Figure 5 presents a flowchart about the main function for the data acquisition process.

An SD card is used to store the measured data locally in the weather station. For this purpose, a module to interface the SD card to the microcontroller was used. This module communicates with the microcontroller using the SPI protocol. A communication block in the microcontroller was programmed as SPI master, whereas the SD card module works as SPI slave. The open source file system FatFs is used to read and write the text files in the SD card. A new text file is created to store all the data acquired on each day. Another text file, called dates.txt, is used to store the starting and end times of acquisition for all the days, as well as the sampling rate. Every time there is new data to save in the SD card, it checks if it is a new day or is it still the same day and acts accordingly. 


\subsection{Wireless Data Transfer}

The BLE network was programmed in the PSoC module using the BLE component, where is placed the design diagram of the PSoC Creator IDE. Though the BLE component, various parameters of the BLE network can be configured, such as the connection interval, the advertising interval and the MTU (Maximum Transmission Unit) size. This component also allows the creation of the GATT (Generic Attribute Profile) services and characteristics that used to transfer the data through the BLE network. The GAP (Generic Access Profile) role of this component was defined as peripheral device, since it will advertise its presence to central device (Android smartphone), which will be responsible for establishing the BLE connection. The peripheral device component was also defined as GATT server, since it will provide the data requested by the GATT client (central device).

The data throughput of the BLE connection was maximized in order to minimize the data transfer time. There are two main BLE parameters that affect the throughput: the maximum packet length (MTU size) and the packet transmission interval (BLE connection interval). The MTU size can be configured to a value between 23 bytes and 512 bytes, whereas the connection interval can be set to a value between $7.5 \mathrm{~ms}$ and $4 \mathrm{~s}$, with higher energy consumption for lower values. Since the data transfer process will not be much frequent, the minimum possible value for the connection interval ( $7.5 \mathrm{~ms}$ ) was chosen, and the MTU size was set to 500 bytes.

A GATT service and its characteristics were then created to accommodate all the data transfer needs. Since the data to be transferred can take up to a few dozens of kilobytes per text file, a characteristic called DataArray, with a type of 16-bit integer array and a length of 250 bytes, was created. With a maximum of 500 bytes to be transferred per packet, this characteristic needs to be read several times. For that, an algorithm to transfer the data was created, as shown in Figure 6. After reading the data from the text file, it is saved in a buffer in the RAM (Random Access Memory). It was also created a characteristic called NumBytes, used to notify the Android device of the amount of data about to be received per packet. Another characteristic, called Next, was also created to inform the GATT server (PSoC device) when the DataArray characteristic was read. After receiving each 500-byte data segment form the buffer, the Android device writes the value ' 1 ' in the Next characteristic, meaning that it is ready to receive the next 500 bytes from the buffer (or up to 500 bytes, in the case of the last data packet). This process is done multiple times until the end of the buffer.

\subsection{Mobile App}

An Android mobile app was developed in order to handle the BLE data transfer and to allow the storage and visualization of the data received from the weather station. The mobile app also allows the configuration of some parameters related to the data acquisition performed by the weather station. The mobile app was developed using the Android Studio IDE.

To use the Bluetooth hardware of the Android device, it was necessary to require some permissions in the Android manifest. It was developed an activity to scan for 

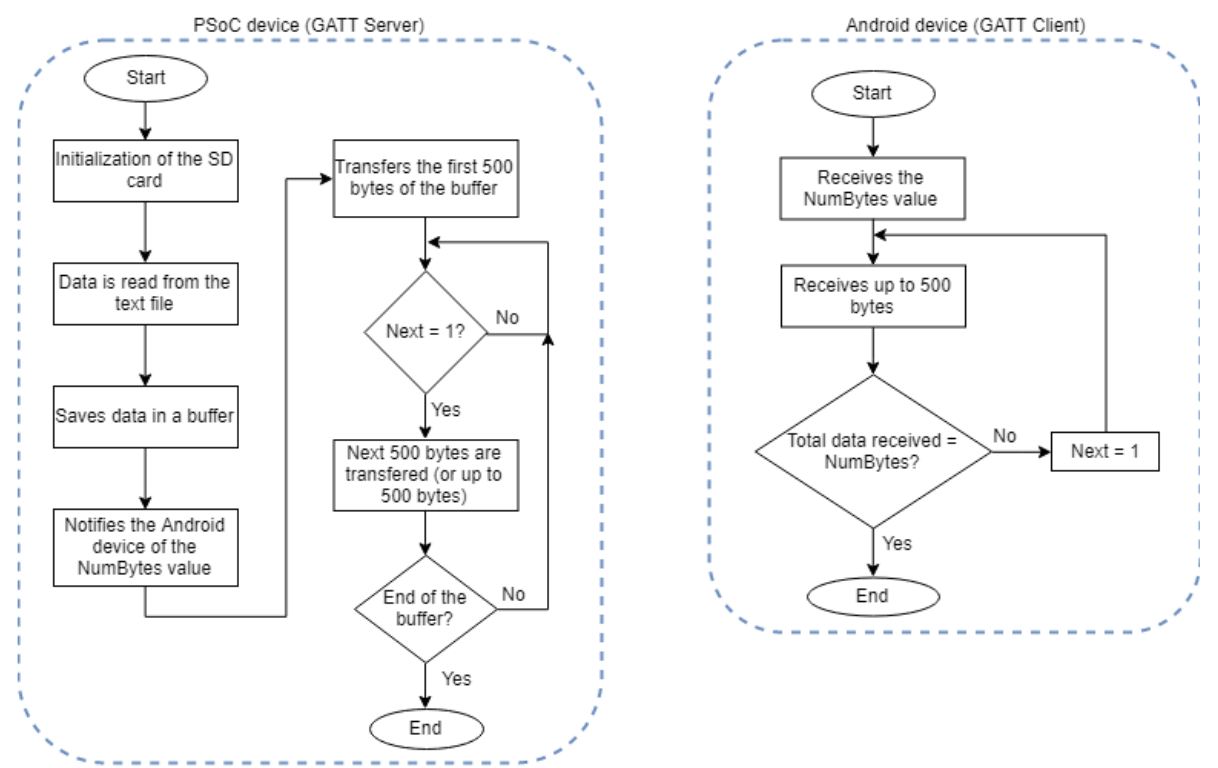

Fig. 6. Developed algorithm for data transfer using BLE.

BLE devices, which displays their names or addresses, as well as their RSSI (Received Signal Strength Indication). After selecting the weather station device from the presented list of BLE devices, the user is asked for the password to access the other features of the app. A service was created to accommodate all the processes related to BLE, including connecting to the GATT server, discovering services, as well as handling the data transfer process.

The mobile app design is then divided into two main fragments. A fragment called HomeFragment is used to display the current values of the data acquired by the weather station in real-time. This screen also allows the configuration of the daily starting and ending data acquisition times, as well as the sampling period for the data acquisition. The other fragment, called DataFragment, is used to transfer the data stored in the weather station. In this screen, the user can select the day from the available dates. It is also possible to enable an option that transfers the data gathered from all the days so far. After transferring the data, it is saved locally in a SQLite database using two tables for this purpose. A ListView is then updated in the screen with the available dates. To visualize the collected data, the user can choose a date from the list, and another activity dedicated to presenting graphs is opened, where the user can select the parameters that he wishes to visualize. This activity was developed using the MPAndroidChart library.

\section{Experimental Results}

This section presents the main results obtained from experimental tests, which are used to validate the implementation of the whole system, ranging from the data acqui- 


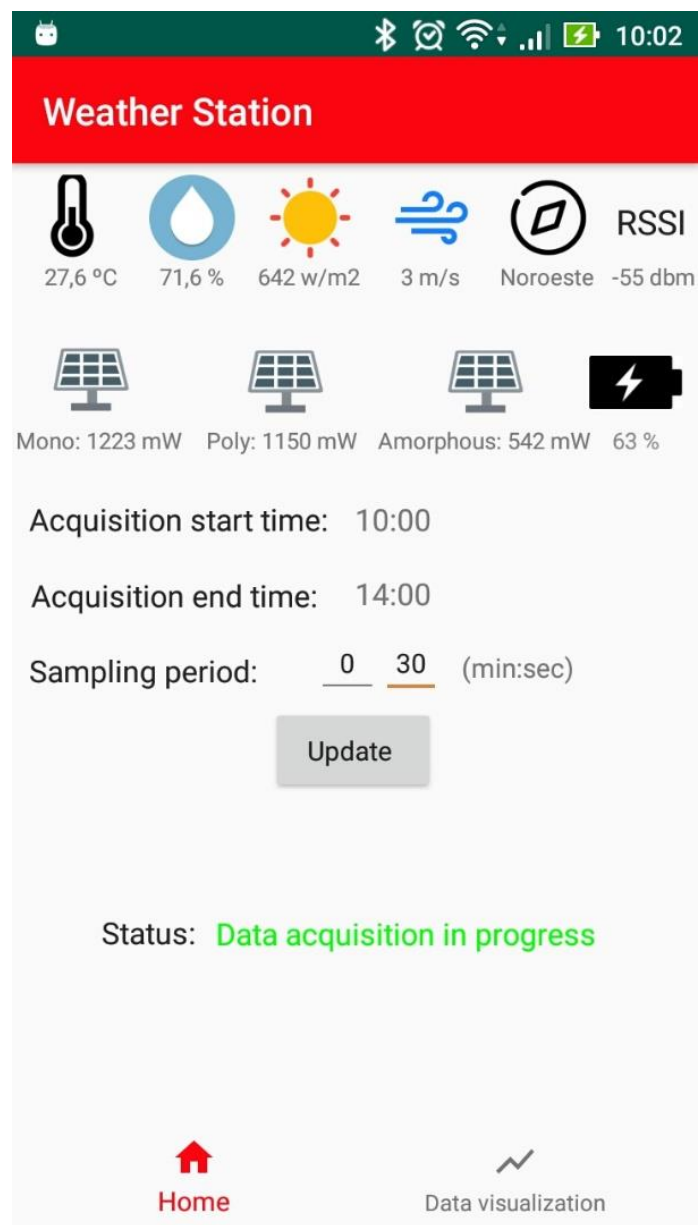

Fig. 7. Home screen of the developed mobile app.

sition in the weather station to the visualization in a smartphone. The developed mobile app has a bottom navigation menu that allows the user to transition between its screens. After connecting to the weather station, the default screen is the home screen, as shown in Figure 7.

This screen allows the user to set the start and ending times of the acquisition, as well as the sampling period (in this case, a fragment dedicated to choosing the hours and minutes will pop up). It is also possible to change the value of the sampling period (the default value is 30 seconds). When the data acquisition starts, the TextView elements that present the last acquired values, from all the weather station sensors, are continuously updated in real-time (top of the screen). The user can also change the start and end times of acquisition, as well as the sampling period, even if there is data being acquired (the new values will be updated for the next day). 


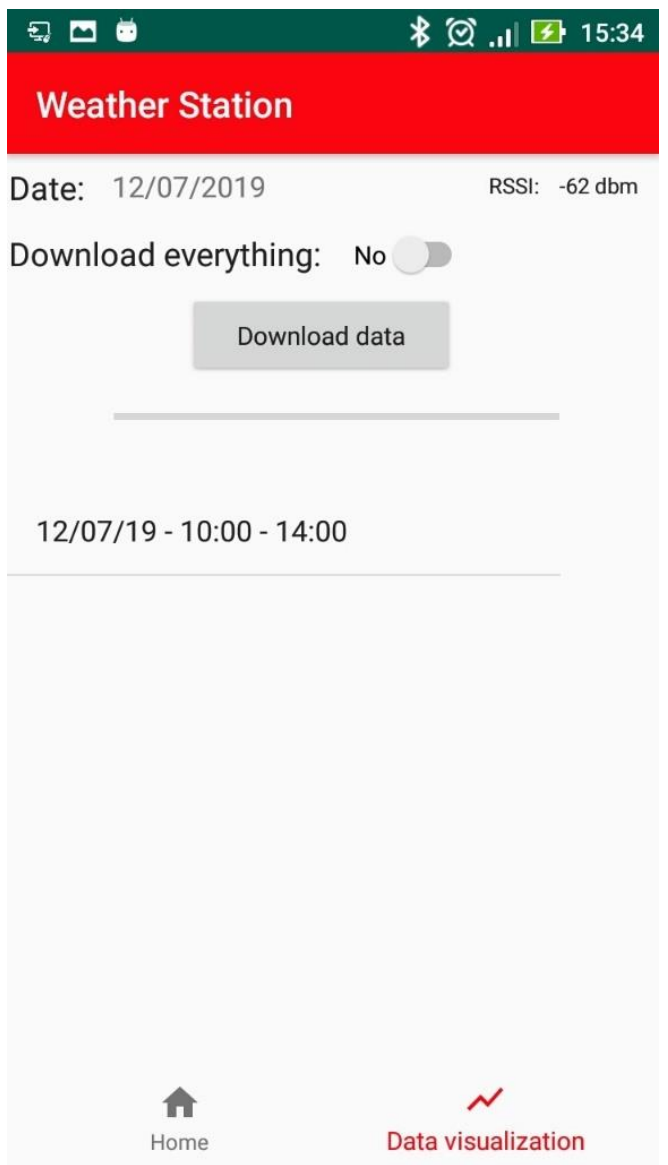

Fig. 8. Data visualization screen of the developed mobile app.

The second screen, shown in Figure 8, is dedicated to download and visualize the weather station data. Here, the user can choose a day to download the respective acquired data, or enable an option that will download the data acquired from all the available days. A progress bar is updated as the data is transferred. The data is stored in a SQLite database, and, after finishing the download, a list is updated in the screen with the dates and the respective starting and ending times.

To visualize the data, the user can tap on one of the elements of the list. This will retrieve the data from the SQLite database and open the activity that allows the user to view the data in charts, as shown in Figure 9. In this screen, the user can choose which information to visualize, by selecting one of the checkboxes. The axes scales with the maximum and minimum values for the chart will automatically change according to the data values. The example shown in Figure 9 presents the temperature values collected by the weather station, obtained between 10 am and 14 pm of July 12, 2019. 


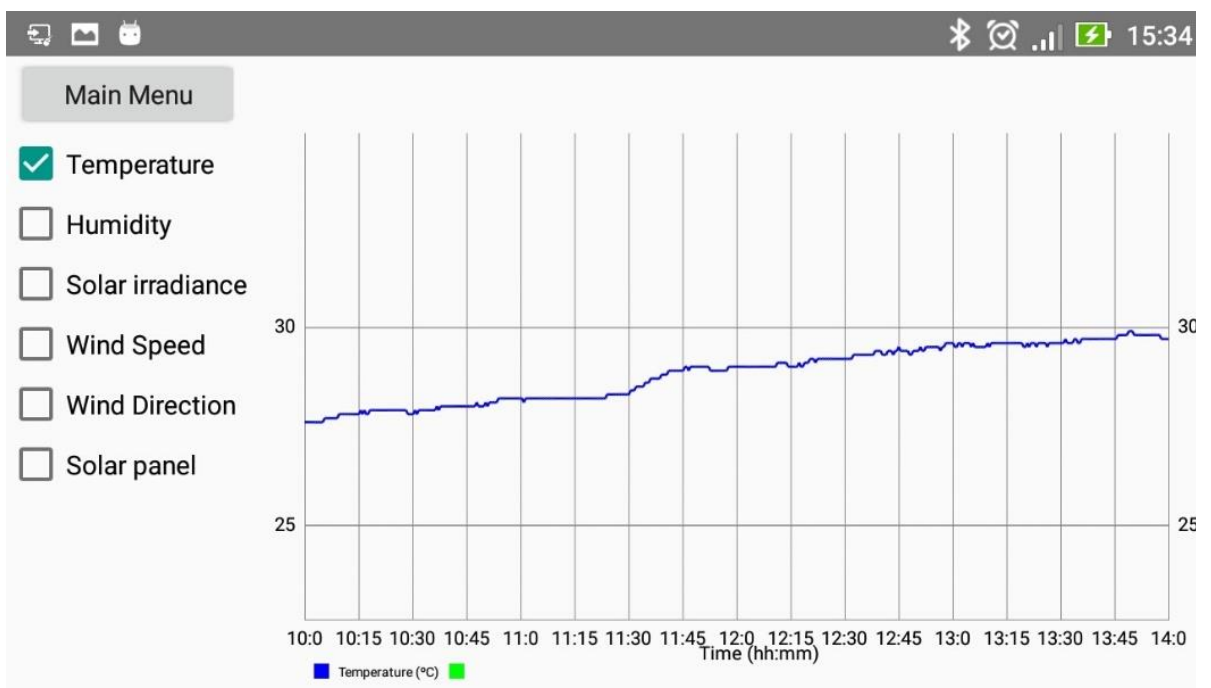

Fig. 9. Screen of the developed mobile app, showing the chart activity that allows the user to visualize the collected data.

\section{Conclusions}

This paper presents the development of a weather station dedicated to renewable energies applications, which also includes a mobile app that provides a user interface and allows data transfer, storage and visualization. The developed system is composed by various sensors used to measure the state of the weather, as well as the power production from the three different technologies of solar photovoltaic modules, installed in the weather station (a module of each type: polycrystalline, monocrystalline and amorphous silicon). The key purpose of using these different solar photovoltaic modules is measuring the produced power for each one of them in order to gauge which technology would be me more suitable for the specific location where the weather location is installed.

All the data acquired is processed and stored locally in the weather station. When necessary, the user can download the data to an Android mobile device through a BLE wireless network connection using the developed mobile app, presented in this paper. This transferred data is stored in a SQLite database and allows the user to visualize it, with the help of dedicated charts for the measured variables.

The system was validated through experimental tests, by acquiring data using all the sensors, storing the information locally in the weather station, transferring the data to an Android smartphone via BLE, storing it in the smartphone, and presenting the data to the user. The starting and ending times of acquisition, as well as the sampling period, can be defined by the user with the developed mobile app. 


\section{References}

1. F. R. Pazheri, M. F. Othman, and N. H. Malik, "A review on global renewable electricity scenario,” Renew. Sustain. Energy Rev., vol. 31, pp. 835-845, 2014.

2. M. Q. Raza, M. Nadarajah, and C. Ekanayake, "On recent advances in PV output power forecast," Sol. Energy, vol. 136, pp. 125-144, 2016.

3. S. Lo Piano and K. Mayumi, "Toward an integrated assessment of the performance of photovoltaic power stations for electricity generation," Appl. Energy, vol. 186, pp. 167174, 2017.

4. P. K. Nayak, S. Mahesh, H. J. Snaith, and D. Cahen, "Photovoltaic solar cell technologies: analysing the state of the art," Nat. Rev. Mater., vol. 4, no. 4, pp. 269-285, 2019.

5. V. Perraki and P. Kounavis, "Effect of temperature and radiation on the parameters of photovoltaic modules," vol. 013102, no. 2016, 2017.

6. C. Ranhotigamage and S. C. Mukhopadhyay, "Field trials and performance monitoring of distributed solar panels using a low-cost wireless sensors network for domestic applications," IEEE Sens. J., vol. 11, no. 10, pp. 2583-2590, 2011.

7. A. Kovacs, A. Nicolcioiu, J. Arhip, and G. Caşu, "Design and implementation of a GPRS remote data logger for weather forecasting," IEEE Int. Conf. Commun., 2014.

8. D. Hortelano, T. Olivares, M. C. Ruiz, C. Garrido-Hidalgo, and V. López, "From sensor networks to internet of things. Bluetooth low energy, a standard for this evolution," Sensors (Switzerland), vol. 17, no. 2, pp. 1-31, 2017.

9. G. R. Hiertz, D. Denteneer, L. Stibor, Y. Zang, X. P. Costa, and B. Walke, "The IEEE 802.11 universe," IEEE Commun. Mag., vol. 48, no. 1, pp. 62-70, 2010.

10. U. Raza, P. Kulkarni, and M. Sooriyabandara, "Low Power Wide Area Networks: An Overview," IEEE Commun. Surv. Tutorials, vol. 19, no. 2, pp. 855-873, 2017.

11. P. Baronti, P. Pillai, V. W. C. Chook, S. Chessa, A. Gotta, and Y. F. Hu, "Wireless sensor networks: A survey on the state of the art and the 802.15.4 and ZigBee standards," Comput. Commun., vol. 30, no. 7, pp. 1655-1695, 2007.

12. Cypress Semiconductor, "CY8CKIT-042-BLE-A Bluetooth Low Energy 4.2 Compliant Pioneer Kit.” [Online]. Available: https://www.cypress.com/documentation/developmentkitsboards/cy8ckit-042-ble-bluetooth-low-energy-42-compliant-pioneer-kit. [Accessed: 09-Jul-2019].

13. Vishay Semiconductors, "bpw34.pdf." [Online]. Available: https://www.vishay.com/docs/81521/bpw34.pdf. [Accessed: 09-Jul-2019].

14. WaveShare, "DHT22 Temperature-Humidity Sensor." [Online]. Available: https://www.waveshare.com/wiki/DHT22_Temperature-Humidity_Sensor.

15. E. Ramsden, Hall-Effect Sensors Theory and Application. Newnes, 2006.

16. B. Subudhi and R. Pradhan, "A comparative study on maximum power point tracking techniques for photovoltaic power systems," IEEE Trans. Sustain. Energy, vol. 4, no. 1, pp. 89-98, 2013. 